\title{
A Hyland-Bernstein approach to the digital control of Pritchard-Salamon systems
}

\author{
Florin Dan Barb and Willem De Koning \\ Faculty of Technical Mathematics and Informatics, Delft University of Technology, \\ P.O. Box 5031, 2600 GA Delft, The Netherlands
}

[Received 14 October 1993]

\begin{abstract}
In this paper we consider the fixed finite-order digital linear-quadratic control of Pritchard-Salamon infinite-dimensional systems with unbounded input and output operators under gaussian disturbances. A set of necessary conditions is given in terms of the solvability of a discrete-time Hyland-Bernstein system of equations (two modified Riccati equations and two modified Lyapunov equations coupled by an projection operator).
\end{abstract}

\section{Introduction}

In [10,11] Pritchard \& Salamon introduced a class of infinite-dimensional systems that allow certain unboundedness in control and observation. Common examples of such systems are linear systems described by PDEs with point and/or boundary control and observation [8], and retarded systems with delays in control and observation [10]. This class has been extensively studied, since it is rich enough to permit one to solve the linear-quadratic (LQ) and $\mathcal{H}^{\infty}$ control problems $[11,12]$. For an overview of the basic results concerning perturbation theory, exponentially stability, and transfer functions of Pritchard-Salamon systems, the reader is referred to the recently announced paper [9].

One of the main assumptions of the so-called direct approach to designing a reduced-order controller is to consider it as having a fixed specified structure and a given fixed order. Thus, the controller parameters are determined by solving a certain optimization problem. Bernstein \& Hyland [6] showed that the set of firstorder necessary conditions for the quadratically optimal continuous-time fixedfinite-order dynamic compensation of infinite-dimensional systems with bounded input and output operators can be transformed into a system of four coupled matrix equations-two modified Riccati equations and two modified Lyapunov equations - coupled by an oblique projection operator which arises naturally from the optimality constraints on the design process. We shall call such a system a Hyland-Bernstein system of equations. The foundations for the continuous-time setting have been developed by Bernstein et al. [5] in the discrete-time case when the plant was assumed to be finite-dimensional. An extension of those results for the case of discrete-time systems with multiplicative white noise can be found [7]. Recently, a complete generalization of the results in [5,7] has been obtained by Barb \& De Koning [4] for infinite-dimensional discrete-time time-varying systems. 
The digital LQ control problem for Pritchard-Salamon systems has been addressed and solved in [3]. This paper is a natural continuation of the work in $[3,4]$. We are concemed with the case when the continuous-time state and the discrete values of the sampled measurement output are corrupted by white gaussian noises. Our goal is to design a discrete-time controller of a specified order, interconnected with the continuous-time plant via a synchronized sampler and zeroth-order holder, such that the effect of the disturbance input on the regulated output, evaluated via the 'classical' LQ gaussian (LQG) function, is minimized over the class of stabilizing compensators. The piecewise constant control is generated from the discrete values of the measured output. The main result of this paper is a set of necessary solvability conditions expressed in terms of a Hyland-Bernstein system of equations.

\section{The fixed-order digital LQG control problem for Pritchard-Salamon systems}

Let us consider three real separable Hilbert spaces $\mathcal{W}, \mathcal{X}$, and $\mathcal{V}$ with continuous dense injections $\mathcal{W} \hookrightarrow \mathcal{X} \hookrightarrow \mathcal{V}$. We consider a $\mathrm{C}_{0} \operatorname{semigroup}\{\Phi(t)\}_{t \in \mathbf{R}_{+}}$on $\mathcal{V}$, and we shall assume that its restriction on $\mathcal{W}$ and $\mathcal{X}$ are also $\mathrm{C}_{0}$ semigroups. $A^{\mathcal{W}}, A^{\mathcal{X}}$, and $A^{\mathcal{V}}$ will denote the infinitesimal generators of $\left\{\Phi^{\mathcal{W}}(t)\right\}_{t \in \mathbf{R}_{+}},\left\{\Phi^{\mathcal{X}}(t)\right\}_{t \in \mathbf{R}_{+}}$, and $\left\{\Phi^{\mathcal{V}}(t)\right\}_{t \in \mathbf{R}_{+}}$on $\mathcal{W}, \mathcal{X}$, and $\mathcal{V}$. The following definition is reported from $[9,11,12]$.

Definition 1 Let $\mathcal{U}$ and $\mathcal{Y}$ be the input and output spaces.

1. An operator $B \in \mathfrak{L}(\mathcal{U}, \mathcal{V})$ is called an admissible input operator for $\Phi(\bullet)$ if there exist $t>0$ and $\alpha>0$ such that $\int_{0}^{t} \Phi^{\mathcal{V}}(t-\tau) B u(\tau) \mathrm{d} \tau \in \mathcal{W}$ and

$$
\left\|\int_{0}^{t} \Phi^{\mathcal{V}}(t-\tau) B u(\tau) \mathrm{d} \tau\right\|_{\mathcal{W}} \leqslant \alpha\|u(\bullet)\|_{\mathrm{L}_{2}(0, t ; \mathcal{L})}
$$

for all $u(\bullet) \in \mathrm{L}_{2}(0, t ; \mathcal{U})$.

2. An operator $C \in \mathfrak{L}(\mathcal{W}, \mathcal{Y})$ is called an admissible output operator for $\Phi(\bullet)$ if there exist $t>0$ and $\beta>0$ such that

$$
\left\|C \Phi^{\mathcal{W}}(\cdot) x\right\|_{L_{2}(0, r ; Y)} \leqslant \beta\|x\|_{\mathcal{V}}
$$

for all $x \in \mathcal{W}$.

3. Let $B \in \mathfrak{L}(\mathcal{U}, \mathcal{V})$ and $C \in \mathfrak{L}(\mathcal{W}, \mathcal{Y})$ be admissible input and output operators for $\Phi(\bullet)$. The system $\Sigma(\Phi(\bullet), B, C, D)$ given by

$$
\Sigma:\left\{\begin{array}{l}
x(t)=\Phi^{\nu}(t) x_{0}+\int_{0}^{t} \Phi^{\mathcal{\nu}}(t-\tau) B u(\tau) \mathrm{d} \tau \\
y(t)=C x(t)+D u(t)
\end{array}\right.
$$

where $x_{0} \in \mathcal{V}, t \geqslant 0$ and $u(\bullet) \in \mathrm{L}_{2}(0, t ; \mathcal{U})$ is called a Pritchard-Salamon system.

Following [9], we assume without loss of generality that the Pritchard-Salamon system is smooth, i.e. there is a continuous dense injection from the domain of $A^{\mathcal{V}}$ into $W$ :

$$
\mathcal{D}\left(A^{\mathcal{V}}\right) \hookrightarrow \mathcal{W}
$$


Consider now three Hilbert spaces with continuous dense injections $\mathcal{W} \hookrightarrow \mathcal{X} \hookrightarrow \mathcal{V}$, and assume that there exist other real separable Hilbert spaces $\mathcal{U}_{1}$ and $\mathcal{Y}_{1}$; and let $B_{1} \in \mathfrak{L}\left(\mathcal{U}_{1}, \mathcal{V}\right), \quad B_{2} \in \mathfrak{Q}\left(\mathbb{R}^{m}, \mathcal{V}\right), \quad C_{1} \in \mathfrak{L}\left(\mathcal{W}, \mathcal{Y}_{1}\right), \quad C_{2} \in \mathfrak{L}\left(\mathcal{W}, \mathbb{R}^{p}\right), \quad D_{11} \in \mathfrak{S}\left(\mathcal{U}_{1}, \mathcal{Y}_{1}\right)$, $D_{12} \in \mathfrak{L}\left(\mathbb{R}^{m}, \mathcal{Y}_{1}\right)$. We consider the smooth Pritchard-Salamon system

$$
\Sigma\left(\Phi(\bullet),\left[B_{1}, B_{2}\right],\left[\begin{array}{c}
C_{1} \\
C_{2}
\end{array}\right],\left[D_{11}, D_{12}\right]\right)
$$

satisfying

$$
\Sigma:\left\{\begin{array}{l}
x(t)=\Phi(t) x_{0}+\int_{0}^{t} \Phi(t-\tau)\left(B_{1} u_{1}(\tau)+B_{2} u_{2}(\tau)\right) \mathrm{d} \tau \\
y_{1}(t)=C_{1} x(t)+D_{11} u_{1}(t)+D_{12} u_{2}(t)
\end{array}\right.
$$

and the discrete-time measurement output equation

$$
y_{2, k}=C_{2} x_{k}+D_{12} u_{3, k} \quad(k=0,1, \ldots) .
$$

In our set-up, $x(t)$ is the state, $u_{1}(t) \in \mathcal{U}_{1}$ is the disturbance input assumed to be white gaussian noise [1], $u_{2}(t) \in \mathbb{R}^{m}$ is the control input, $y_{1}(t) \in \mathcal{Y}_{1}$ the regulated output, and $y_{2, k} \in \mathbb{R}^{P}$ the sampled measured output corrupted by a white gaussian noise $u_{3, k} \in \mathbb{R}^{p}$. We assume that the initial state $x_{0} \in \mathcal{W}$ is a random variable independent of the noise $u_{1}$.

Let now $\mathbb{R}^{n \times n}$ be the controller state space, and suppose that we have a controller $\Sigma(K, L, M)$ of the form

$$
\Sigma_{\mathrm{K}}:\left\{\begin{array}{l}
\zeta_{k+1}=K \zeta_{k}+L \eta_{k}, \\
\xi_{k}=M \zeta_{k}
\end{array}\right.
$$

where $\zeta \in \mathbb{R}^{n \times n}$ is the controller state, $\eta \in \mathbb{R}^{p}$ is the controller input and $\xi \in \mathbb{R}^{m}$ is the controller output. We want to interconnect the system and the discrete-time controller via $\mathrm{A} / \mathrm{D}$ and $\mathrm{D} / \mathrm{A}$ devices, and to perform a digital control. For this we define the sample and zeroth-order hold operators with period $T$ as

$$
\begin{aligned}
S: \mathbb{C}(0, \infty ; \mathcal{X}) \rightarrow \mathcal{X}^{\mathbf{N}}, & x_{k}=S x(t)=x(k T), \\
H: \mathcal{X}^{\mathbf{N}} \rightarrow \mathfrak{C}(0, \infty ; \mathcal{X}), & u(t)=H u_{k}=u_{k},
\end{aligned}
$$

where $\mathbb{C}(0, \infty ; \mathcal{X})$ stands for the space of $\mathcal{X}$-valued piecewise continuous functions bounded on compact subsets of $[0, \infty)$, and $\mathcal{X}^{\mathbf{N}}$ is the space of $\mathcal{X}$-valued sequences defined on the set of integers. We assume that the hold operator is synchronized in time with the sampling operator. Clearly, we want to make sense of the following feedback interconnection:

$$
\eta_{k}=y_{2, k} \quad(k=0,1, \ldots), \quad u_{2}(t)=\xi_{k} \quad(k T \leqslant t<(k+1) T ; k=0,1, \ldots) .
$$

Our goal is to design a controller $\Sigma_{\mathrm{K}}=\Sigma(K, L, M)$ meeting the following specifications.

- Optimality: The influence of the disturbance input $u_{1}$ on the regulated output $y_{1}$ is minimized in the sense that the LQG cost function

$$
J(K, L, M)=\lim _{t \rightarrow \infty} \frac{1}{t} \mathrm{E} \int_{0}^{t}\left\langle y_{1}(\tau), y_{1}(\tau)\right\rangle \mathrm{d} \tau
$$

is minimized over the set of stabilizing controllers $\Sigma_{\mathbf{K}}$. 
- Stability: The linear system from $u_{1}$ to $y_{1}$ defines an exponentially stable evolution.

In order to guarantee that $(2.1)$ is independent of the internal realization of the controller, we shall focus on the following class of controllers

$$
\mathcal{A}=\left\{\Sigma_{\mathrm{K}} \text { stabilizing } \mid(K, L, M) \text { is minimal }\right\} \text {. }
$$

\section{The equivalent discrete-time control problem}

In this section we give the main results on lifting a continuous-time periodic system to a discrete-time time invariant one. Our basic references are $[2,13]$. Let us focus on state-space formulae for lifted systems. Consider the continuous-time system $\Sigma(\Phi(\bullet), B, C, D)$ on a real separable Hilbert space $\mathcal{X}$ given by

$$
\Sigma: \quad\left\{\begin{array}{l}
x^{\prime}(t)=A x(t)+B u(t) \\
y(t)=C x(t)+D u(t)
\end{array}\right.
$$

where $\Phi(\bullet)$ is a strongly continuous semigroup generated by $A$. Let $\mathcal{U}$ and $\mathcal{Y}$ be the real separable Hilbert spaces of the inputs and outputs respectively. The state equation can be re-written as

$$
x(k T+t)=\Phi(t) x(k T)+\int_{0}^{t} \Phi(t-\tau) B u(k T+\tau) \mathrm{d} \tau .
$$

Define the lifting operator

$$
\Omega: \mathrm{L}_{2}(0, \infty ; \mathcal{X}) \rightarrow \mathrm{\ell}_{2}\left(0, \infty ; \mathrm{L}_{2}(0, T ; \mathcal{X})\right): x \mapsto \Omega x
$$

by $\hat{x}=\Omega x$ where $\hat{x}_{k}(\bullet)=x(k T+\bullet)$. Notice that $x_{k}=x(k T)=\hat{x}_{k}(0)$. Then $\hat{x}_{k}:[0, T) \rightarrow \mathrm{L}_{2}(0, T ; \mathcal{X})$ given by

$$
\hat{x}_{k}(\bullet)=\Phi(\bullet) \hat{x}_{k}(0)+\int_{0}^{\bullet} \Phi(\bullet-\tau) B \hat{u}_{k}(\tau) \mathrm{d} \tau
$$

is a well defined application, and we shall call $\hat{x}=\left\{\hat{x}_{k}(\bullet)\right\}_{k>0} \in \ell_{2}\left(0, \infty ; \mathrm{L}_{2}(0, T ; \mathcal{X})\right)$ given by (3.2) the lifted state corresponding to $x \in \mathrm{L}_{2}(0, \infty ; \mathcal{X})$. Notice that the state equation (3.1) has an equivalent form given by

$$
x_{k+1}=\hat{A} x_{k}+\hat{B} \hat{u}_{k}
$$

with

$$
\hat{A}=\Phi(T), \quad \hat{B} \hat{u}_{k}=\int_{0}^{T} \Phi(T-\tau) B \hat{u}_{k}(\tau) \mathrm{d} \tau
$$

and from (3.3) and (3.1) we finally obtain

$$
\begin{aligned}
\hat{y}_{k} & =C \hat{x}_{k}+D \hat{u}_{k}=C\left(\Phi(\bullet) x_{k}+\int_{0}^{\bullet} \Phi(\bullet-\tau) B \hat{u}_{k}(\tau) \mathrm{d} \tau\right)+D \hat{u}_{k} \\
& =C \Phi(\bullet) x_{k}+C \int_{0}^{\bullet} \Phi(\bullet-\tau) B \hat{u}_{k}(\tau) \mathrm{d} \tau+D \hat{u}_{k} .
\end{aligned}
$$


Define now

$$
\hat{C}=C \Phi(\bullet), \quad \hat{D} \hat{u}_{k}=C \int_{0}^{\bullet} \Phi(\bullet-\tau) B \hat{u}_{k}(\tau) \mathrm{d} \tau+D \hat{u}_{k} .
$$

Then the original system (3.1) admits an equivalent representation

$$
\mathrm{s} x=\hat{A} x+\hat{B} \hat{u}, \quad \hat{y}=\hat{C} x+\hat{D} \hat{u},
$$

where $\hat{A}, \hat{B}, \hat{C}$, and $\hat{D}$ are defined above, and where $x \in \ell_{2}(0, \infty ; \mathcal{X})$, $\hat{u} \in \ell_{2}\left(0, \infty ; \mathrm{L}_{2}(0, T ; \mathcal{U})\right)$, and $\hat{y} \in \ell_{2}\left(0, \infty ; \mathrm{L}_{2}(0, T ; \mathcal{Y})\right)$; here $s$ denotes the forward shift operator: $s x(\bullet)=x(\bullet+1)$.

Consider the smooth Pritchard-Salamon system $\Sigma\left(\Phi(\bullet),\left[B_{1}, B_{2}\right], C_{1},\left[D_{11}, D_{12}\right]\right)$ with respect to $\mathcal{W} \hookrightarrow \mathcal{X} \hookrightarrow \mathcal{V}$; as in [3], we shall restrict to the case when we have finite many actuators and sensors, $p$ and $m$ respectively. This condition is essential from the engineering point of view. Let us apply the lifting technique under the assumption that $u_{2}$ is obtained in a piecewise fashion from the sampled measured output $y_{2}$. The lifted state equation written for the sampling instants is

$$
x_{k+1}=\hat{\Phi} x_{k}+\hat{\Gamma}_{1} \hat{u}_{1, k}+\Gamma_{2} u_{2, k},
$$

with

$$
\begin{gathered}
\hat{\Phi}=\Phi^{\mathcal{W}}(T), \quad \hat{\Gamma}_{1} \hat{u}_{1, k}=\int_{0}^{T} \Phi^{\mathcal{W}}(T-\tau) B_{1} \hat{u}_{1, k}(\tau) \mathrm{d} \tau \\
\Gamma_{2}=\int_{0}^{T} \Phi^{\mathcal{W}}(T-\tau) B_{2} \mathrm{~d} \tau
\end{gathered}
$$

and the equation for the regulated output is

$$
\begin{aligned}
\hat{y}_{1, k}= & C_{1} x_{k}+D_{11} \hat{u}_{1, k}+D_{12} u_{2, k} \\
= & C_{1} \Phi^{\mathcal{W}}(\cdot) x_{k}+C_{1} \int_{0}^{\bullet} \Phi^{\mathcal{W}}(\cdot-\tau) B_{1} \hat{u}_{1, k}(\tau) \mathrm{d} \tau+\left(C_{1} \int_{0}^{\bullet} \Phi^{\mathcal{W}}(\cdot-\tau) B_{2} \mathrm{~d} \tau\right) u_{2, k} \\
& +D_{11} \hat{u}_{1, k}+D_{12} u_{2, k}=\hat{C}_{1} x_{k}+\hat{D}_{11} \hat{u}_{1, k}+D_{12}^{\mathrm{e}} u_{2, k},
\end{aligned}
$$

with

$$
\begin{gathered}
\hat{C}_{1}=C_{1} \Phi^{\mathcal{W}}(\bullet), \\
\hat{D}_{11} \hat{u}_{1, k}=C_{1} \int_{0}^{\bullet} \Phi^{\mathcal{W}}(\bullet-\tau) B_{1} \hat{u}_{1, k}(\tau) \mathrm{d} \tau+D_{11} \hat{u}_{1, k}, \\
D_{12}^{\mathrm{e}}=C_{1} \int_{0}^{\bullet} \Phi^{\mathcal{W}}(\bullet-\tau) B_{2} \mathrm{~d} \tau+D_{12} .
\end{gathered}
$$

We consider the sampling process as being affected by a discrete-time white gaussian noise $u_{3} \in \ell_{2}\left(0, \infty ; \mathbb{R}^{p}\right)$ :

$$
y_{2, k}=C_{2} x_{k}+D_{21} u_{3, k}
$$


With this, we have obtained the following discrete-time representation for the original system:

$$
\begin{gathered}
s x=\hat{\Phi} x+\left[\hat{\Gamma}_{1}, 0\right]\left[\begin{array}{l}
\hat{u}_{1} \\
u_{3}
\end{array}\right]+\Gamma_{2} u_{2}, \\
\hat{y}_{1}=\hat{C}_{1} x+\left[\hat{D}_{11}, 0\right]\left[\begin{array}{l}
\hat{u}_{1} \\
u_{3}
\end{array}\right]+D_{12}^{\mathrm{e}} u_{2}, \quad y_{2}=C_{2} x+\left[0, D_{21}\right]\left[\begin{array}{l}
\hat{u}_{1} \\
u_{3}
\end{array}\right],
\end{gathered}
$$

where

$$
\begin{aligned}
& x \in \ell_{2}(0, \infty ; \mathcal{W}), \quad \hat{u}_{1} \in \ell_{2}\left(0, \infty ; L_{2}\left(0, T ; \mathcal{U}_{1}\right)\right), \\
& \hat{y}_{1} \in \ell_{2}\left(0, \infty ; L_{2}\left(0, \infty ; \mathcal{Y}_{1}\right)\right), \quad y_{2} \in \ell_{2}\left(0, \infty ; \mathbb{R}^{p}\right), \quad u_{3} \in \ell_{2}\left(0, \infty ; \mathbb{R}^{m}\right),
\end{aligned}
$$

LEMMA 1 The operators in (3.4) satisfy the boundedness conditions

$$
\begin{array}{cc}
\hat{\Phi} \in \mathfrak{L}(\mathcal{W}), \quad \hat{\Gamma}_{1} \in \mathfrak{L}\left(\mathrm{L}_{2}\left(0, T ; \mathcal{U}_{1}\right), \mathcal{W}\right), \quad \Gamma_{2} \in \mathfrak{I}\left(\mathbb{R}^{m}, \mathcal{W}\right), \\
\hat{C}_{1} \in \mathfrak{L}\left(\mathcal{W}, \mathrm{L}_{2}\left(0, T ; \mathcal{Y}_{1}\right)\right), & C_{2} \in \mathfrak{L}\left(\mathcal{W}, \mathbb{R}^{p}\right), \\
\hat{D}_{11} \in \mathfrak{I}\left(\mathrm{L}_{2}\left(0, T ; \mathcal{U}_{1}\right), \mathrm{L}_{2}\left(0, T ; \mathcal{Y}_{1}\right)\right), & D_{12}^{e} \in \mathfrak{L}\left(\mathbb{R}^{m}, \mathrm{~L}_{2}\left(0, T ; \mathcal{Y}_{1}\right)\right) .
\end{array}
$$

Proof. It is sufficient to prove that $\hat{\Gamma}_{1} \in \mathfrak{Q}\left(\mathrm{L}_{2}\left(0, T ; \mathcal{U}_{1}\right), \mathcal{W}\right)$ and $\Gamma_{2} \in \mathfrak{Q}\left(\mathbb{R}^{m}, \mathcal{W}\right)$; the rest follows immediately from the definitions of $\hat{C}_{1}, \hat{D}_{11}$, and $D_{12}^{\mathrm{e}}$. Let us write the condition for $B_{1}$ to be admissible on the time interval $[0, T)$. We obtain

$$
\left\|\int_{0}^{T} \Phi^{\mathcal{W}}(T-\tau) B_{1} u_{1}(\tau) \mathrm{d} \tau\right\|_{\mathcal{W}} \leqslant \alpha\left\|u_{1}\right\|_{L_{2}\left(0, T ; \mathcal{H}_{1}\right)} .
$$

which yields the boundedness condition on $\hat{\Gamma}_{1}$. The same manipulation can be done to show that $\Gamma_{2}$ is bounded.

We are led to the main result of this section.

THEOREM 1 The following hold.

1. Let

$$
\Sigma\left(\Phi(\bullet),\left[B_{1}, B_{2}\right],\left[\begin{array}{l}
C_{1} \\
C_{2}
\end{array}\right],\left[D_{11}, D_{12}\right]\right)
$$

be a smooth Pritchard-Salamon system with respect to $\mathcal{W} \hookrightarrow \mathcal{X} \hookrightarrow \mathcal{V}$. The discretetime controller $\Sigma_{\mathrm{K}}=\Sigma(K, L, M)$, with $(K, L, M) \in \mathbb{R}^{n \times n} \times \mathbb{R}^{n \times m} \times \mathbb{R}^{p \times n}$, given by

$$
\Sigma_{\mathbf{K}}:\left\{\begin{array}{l}
\zeta_{k+1}=K \zeta_{k}+L y_{2, k}, \\
\xi_{k}=M \zeta_{k}+N u_{2, k},
\end{array}\right.
$$

where $N \in \mathbb{R}^{p \times m}$ is a given constant matrix, is a solution to the digital LQG control problem if and only if, for each $t \in[0, \infty)$, it is a solution to the following 
discrete-time LQG control problem:

$$
\text { minimize } \hat{J}(K, L, M)(t)=\lim _{k \rightarrow \infty} \frac{1}{k} \mathrm{E} \sum_{i=0}^{k-1}\left\langle\hat{y}_{1, i}(t), \hat{y}_{1, i}(t)\right\rangle,
$$

over the set of all stabilizing $\Sigma_{\mathrm{K}}$,

for

$$
\begin{gathered}
\mathrm{s} x=\hat{\Phi} x+\left[\hat{\Gamma}_{1}, 0\right]\left[\begin{array}{l}
\hat{u}_{1} \\
u_{3}
\end{array}\right]+\Gamma_{2} u_{2, k}, \\
\hat{y}_{1}=\hat{C}_{1} x+\left[\hat{D}_{11}, 0\right]\left[\begin{array}{l}
\hat{u}_{1} \\
u_{3}
\end{array}\right]+D_{12}^{\mathrm{e}} u_{2}, \quad y_{2}=C_{2} x+\left[0, D_{21}\right]\left[\begin{array}{l}
\hat{u}_{1} \\
u_{3}
\end{array}\right],
\end{gathered}
$$

where

$$
\begin{gathered}
\hat{\Phi}=\Phi^{\mathcal{W}}(T), \\
\hat{\Gamma}_{1} \hat{u}_{1, k}=\int_{0}^{T} \Phi^{\mathcal{W}}(T-\tau) B_{1} \hat{u}_{1, k}(\tau) \mathrm{d} \tau, \quad \Gamma_{2}=\int_{0}^{T} \Phi^{\mathcal{W}}(T-\tau) B_{2} \mathrm{~d} \tau \\
\hat{C}_{1}=C_{1} \Phi^{\mathcal{W}}(\bullet), \\
\hat{D}_{11} \hat{u}_{1, k}=C_{1} \int_{0}^{\bullet} \Phi^{\mathcal{W}}(\bullet-\tau) B_{1} \hat{u}_{1, k}(\tau) \mathrm{d} \tau+D_{11} \hat{u}_{1, k}, \\
D_{12}^{\mathrm{e}}=C_{1} \int_{0}^{\bullet} \Phi^{\mathcal{W}}(\bullet-\tau) B_{2} \mathrm{~d} \tau+D_{12} .
\end{gathered}
$$

2. $\frac{1}{T} \int_{0}^{T} \hat{J}_{\mathrm{m}}(\tau) \mathrm{d} \tau=J_{\mathrm{m}}$, where

$$
\hat{J}_{\mathrm{m}}(t)=\min _{\Sigma_{\mathbf{K}} \text { stabilizang }} \hat{J}(K, L, M)(t), \quad J_{\mathrm{m}}=\min _{\Sigma_{\mathbf{K}} \text { stabulizing }} J(K, L, M) .
$$

Proof. Let us proceed by proving the second item of Theorem 1. Calculate

$$
\begin{aligned}
J_{\mathrm{m}} & =\min _{\Sigma_{\mathrm{k}}} \lim _{\text {stabilizing }} \frac{1}{t \rightarrow \infty} \mathrm{E} \int_{0}^{t}\left\langle y_{1}(\tau), y_{1}(\tau)\right\rangle \mathrm{d} \tau \\
& =\min _{\Sigma_{\mathrm{K}}} \lim _{\text {stabilizing }} \frac{1}{k \rightarrow \infty} \mathrm{E} \sum_{i=0}^{k-1} \int_{i T}^{(i+1) T}\left\langle y_{1}(i T+\tau), y_{1}(i T+\tau)\right\rangle \mathrm{d} \tau \\
& =\min _{\Sigma_{\mathrm{k}}} \text { stabilizing } \frac{1}{T} \lim _{k \rightarrow \infty} \frac{1}{k} \mathrm{E} \sum_{i=0}^{k-1} \int_{0}^{T}\left\langle\hat{y}_{1, i}(\tau), \hat{y}_{1, i}(\tau)\right\rangle \mathrm{d} \tau \\
& =\frac{1}{T} \int_{0}^{T}\left(\min _{\Sigma_{\mathrm{k}}} \min _{\text {stabilizing }} \lim _{k \rightarrow \infty} \frac{1}{k} \mathrm{E} \sum_{i=0}^{k-1}\left\langle\hat{y}_{1}(i), \hat{y}_{1}(i)\right\rangle\right)(\tau) \mathrm{d} \tau \\
& =\frac{1}{T} \int_{0}^{T} \hat{J}_{\mathrm{m}}(\tau) \mathrm{d} \tau .
\end{aligned}
$$

Consider

$$
y_{1} \in \mathrm{L}_{2}\left(0, \infty ; \mathcal{Y}_{1}\right), \quad \hat{y}_{1} \in \ell_{2}\left(0, \infty ; \mathrm{L}_{2}\left(0, T ; \mathcal{Y}_{1}\right)\right)
$$

If $\Sigma_{\mathrm{K}}=\Sigma(K, L, M)$ solves the digital control problem, then $y_{1}$ defines an 
exponentially stable evolution. But $\hat{y}_{1}$ was obtained from $y_{1}$ via lifting, which implies

$$
\left\|\hat{y}_{1}\right\|_{\ell_{2}\left(\mathbf{N} ; L_{2}\left(0, T ; \mathcal{Y}_{1}\right)\right)}=\left\|y_{1}\right\|_{L_{2}\left(0, \infty ; \mathcal{Y}_{1}\right)} \text {, }
$$

which in turn implies that $\hat{y}_{1}$ defines a power-stable evolution. Hence, if $\Sigma_{\mathrm{K}}=\Sigma(K, L, M)$ stabilizes the continuous-time system (i.e. the linear system from $u_{1}$ to $y_{1}$ defines an exponentially stable evolution), then it achieves power stability for the lifted system (i.e. the linear system from $\hat{u}_{1}$ to $\hat{y}_{1}$ defines a power-stable evolution). The converse is also true, since the lifting operator is a linear isometrical application of spaces (see [2]). As for optimality, it is obvious (from a simple inspection of equation (3.6)) that, if $J(K, L, M)$ is minimized, then $\hat{J}(K, L, M)$ is also minimized, and the proof is complete.

\section{The Hyland-Bernstein control result for infinite-dimensional discrete-time systems}

In this section, we give the main Hyland-Bernstein type of result on fixed finite-order control of infinite-dimensional discrete-time systems. We apply it to the lifted Pritchard-Salamon system, obtaining in this way a solution to (3.5) and hence a solution to the digital LQG control of systems in the Pritchard-Salamon class. Our starting point is the following $\ell_{2}$-optimal control problem, which has been completely solved for the general case of time-varying discrete-time systems in [4].

Let $\left(\Phi, \Gamma_{1}, \Gamma_{2}, C_{1}, C_{2}, D_{12}, D_{21}\right)$ denote the linear discrete-time infinite-dimensional system

$$
\Sigma: \quad\left\{\begin{array}{l}
\mathrm{s} x=\Phi x+\Gamma_{1} w+\Gamma_{2}, \\
z=C_{1} x+D_{12} u \\
y=C_{2} x+D_{21} w
\end{array}\right.
$$

on the real separable Hilbert space $\mathcal{X}$, where $\Phi \in \mathfrak{L}(\mathcal{X}), \quad \Gamma_{1} \in \mathfrak{S}\left(\mathcal{U}_{1}, \mathcal{X}\right)$, $\Gamma_{2} \in \mathfrak{L}\left(\mathbb{R}^{m}, \mathcal{X}\right), \quad C_{1} \in \mathfrak{L}\left(\mathcal{X}, \mathcal{Y}_{\infty}\right), \quad C_{2} \in \mathfrak{L}\left(\mathcal{X}, \mathbb{R}^{\vee}\right), \quad D_{12} \in \mathfrak{L}\left(\mathbb{R}^{m}, \mathcal{Y}_{1}\right), \quad$ and $D_{21} \in \mathfrak{L}\left(\mathcal{U}_{1}, \mathbb{R}^{p}\right)$. Here $x, u, w, z$, and $y$ stand for the state, control input, exogenous input, regulated output, and measured output. Let $(K, L, M)$ be a discrete-time $n$ dimensional compensator:

$$
\Sigma:\left\{\begin{array}{l}
\mathrm{s} \xi=K \xi+L y, \\
u=M \xi
\end{array}\right.
$$

where $K \in \mathbb{R}^{n \times n}, L \in \mathbb{R}^{p \times n}$, and $M \in \mathbb{R}^{n \times m}$, with $\xi_{k_{0}}=\xi_{0}$. The closed-loop system, $(\tilde{\Phi}, \tilde{\Gamma}, \tilde{C})$, has its dynamics governed by

$$
\tilde{\Sigma}: \quad\left\{\begin{array}{l}
\mathbf{s} \tilde{x}=\tilde{\Phi} \tilde{x}+\tilde{\Gamma} w \\
z=\tilde{C} \tilde{x}
\end{array}\right.
$$

where $\tilde{\Phi} \in \mathfrak{L}(\tilde{\mathcal{X}}), \tilde{\Gamma} \in \mathfrak{L}\left(\mathcal{U}_{1}, \tilde{\mathcal{X}}\right)$, and $\tilde{C} \in \mathfrak{L}\left(\tilde{\mathcal{X}}, \mathcal{Y}_{1}\right)$, with

$$
\tilde{\Phi}=\left[\begin{array}{cc}
\Phi & \Gamma_{2} M \\
L C_{2} & K
\end{array}\right], \quad \tilde{\Gamma}=\left[\begin{array}{c}
\Gamma_{1} \\
L D_{21}
\end{array}\right], \quad \tilde{C}=\left[C_{1}, D_{12} M\right]
$$


and $\tilde{x}=(x, \xi)$ is the augmented state in $\tilde{\mathcal{X}}=\mathcal{X} \oplus \mathbb{R}^{\backslash}$ which is a real separable Hilbert space under the inner product $\left\langle\tilde{x}_{1}, \tilde{x}_{2}\right\rangle=\left\langle x_{1}, x_{2}\right\rangle+\xi_{1}^{\top} \xi_{2}$ for any $\tilde{x}_{i}=\left(x_{i}, \xi_{i}\right)$ $(i=1,2)$. The closed-loop system is called internally stable if $\tilde{\Phi}$ is power-stable on $\tilde{\mathcal{X}}$. Since our approach is a stochastic one, we shall consider that $w$ is a gaussian zero-mean white noise signal. We consider the initial state to be a random variable which is independent of the noise. Then we can interpret $\Gamma_{1} \Gamma_{1}^{*}, D_{21} D_{21}^{*}, C_{1}^{*} C_{1}$, and $D_{12}^{*} D_{12}$ as being the intensity of the process noise, measurement noise, and state and control weighting operators respectively. We assign to the system the cost function

$$
J(K, L, M)=\lim _{k \rightarrow \infty} \frac{1}{k} \mathrm{E} \sum_{i=0}^{k-1}\left(z_{i}, z_{i}\right\rangle .
$$

The control problem we address is the following. Given the linear discrete-time evolution system $\left(\Phi, \Gamma_{1}, \Gamma_{2}, C_{1}, C_{2}, D_{12}, D_{21}\right)$, find an $n$-dimensional time-varying discrete-time compensator $(F, K, L)$ such that:

- the linear discrete-time evolution system from $w$ to $z$ is internally stable

- the cost function $J(K, L, M)$ defined by (4.1) is minimized over all internally stabilizing $(F, K, L)$.

Controllers belonging to the admissible class

$$
\mathcal{A}_{+}=\{(K, L, M) \mid \tilde{\Phi} \text { is power-stable },(K, L, M) \text { is minimal }\}
$$

guarantee that the cost function is finite, independent of the initial conditions, and independent of the internal realization of the controller. Define the controllability and observability gramians of the closed-loop system as the unique positive semidefinite solutions to the Lyapunov equations

$$
\tilde{P}=\tilde{\Phi}^{*} \tilde{P} \tilde{\Phi}+\tilde{C}^{*} \tilde{C}, \quad \tilde{Q}=\tilde{\Phi} \tilde{Q} \tilde{\Phi}^{*}+\tilde{\Gamma} \tilde{\Gamma}^{*},
$$

and partition them as

$$
\tilde{P}=\left[\begin{array}{cc}
P_{1} & P_{12} \\
P_{12}^{*} & P_{2}
\end{array}\right], \quad \tilde{Q}=\left[\begin{array}{cc}
Q_{1} & Q_{12} \\
Q_{12}^{*} & Q_{2}
\end{array}\right]
$$

Define also $P=P_{1}-P_{12} P_{2}^{-1} P_{12}^{*}$ and $Q=Q_{1}-Q_{12} Q_{2}^{-1} Q_{12}^{*}$. The following assumptions are made on the plant: $\Gamma_{1} \Gamma_{1}^{*}$ and $C_{1}^{*} C_{1}$ are nuclear operators, while $D_{21} D_{21}^{*}+C_{2} Q C_{2}^{*}$ and $D_{12}^{*} D_{12}+\Gamma_{2}^{*} P \Gamma_{2}$ are boundedly invertible. Before stating the main result of this paper, we shall state the discrete-time counterpart of Lemma 3.1 from [6].

Lemma 2 Let $\bar{Q}$ and $\bar{P}=$ be two bounded operators on the real separable Hilbert space $\mathcal{X}$ having finite rank and which are nonnegative definite. Then $\bar{Q} \bar{P}$ is nonnegative semisimple. Furthermore, if rank $\bar{Q} \bar{P}=n$, then there exist $G$ and $\Lambda$ bounded from $\mathcal{X}$ to $\mathbb{R}^{n}$ and an positive semi-simple $n \times n$ matrix $M$ such that

$$
\bar{Q} \bar{P}=G^{*} M \Lambda, \quad \Lambda G^{*}=\mathrm{I}_{n} .
$$

We shall call this the $(G, M, \Lambda)$ factorization of $\bar{Q} \bar{P}$. 
The main result of this section is the following.

Theorem 2 Suppose that $(K, L, M)$ represents a solution to the $\ell^{2}$-optimal discretetime control problem. Then there exist non-negative $Q, P, \bar{Q}, \bar{P} \in \mathcal{L}(\mathcal{X})$ such that $(K, L, M)$ is given by

$$
\begin{aligned}
K= & \Lambda\left[\Phi-\left(\Phi Q C_{2}^{*}+\Gamma_{1} D_{21}^{*}\right)\left(C_{2} Q C_{2}^{*}+D_{21} D_{21}^{*}\right)^{-1} C_{2}\right] \\
& -\Gamma_{2}\left(\Gamma_{2}^{*} P \Gamma_{2}+D_{12}^{*} D_{12}\right)^{-1}\left(\Gamma_{2}^{*} P \Phi+D_{12}^{*} C_{1}\right) G^{*} \\
L= & \Lambda\left(\Phi Q C_{2}^{*}+\Gamma_{1} D_{21}^{*}\right)\left(C_{2} Q C_{2}^{*}+D_{21} D_{21}^{*}\right)^{-1} \\
M= & \left(\Gamma_{2}^{*} P \Gamma_{2}+D_{12}^{*} D_{12}\right)^{-1}\left(\Gamma_{2}^{*} P \Phi+D_{12}^{*} C_{1}\right) G^{*}
\end{aligned}
$$

for some $(G, M, \Lambda)$-factorization of $\hat{Q} \hat{P}$, and such that, if $\tau=G^{*} \Lambda$, then $\operatorname{rank} \hat{Q}=\operatorname{rank} \hat{P}=\operatorname{rank}(\hat{Q} \hat{P})=n$ and the following conditions are satisfied:

$$
\begin{aligned}
& Q=\Phi Q \Phi^{*}+\Gamma_{1} \Gamma_{1}^{*} \\
& -\left(\Phi Q C_{2}^{*}+\Gamma_{1} D_{21}^{*}\right)\left(C_{2} Q C_{2}^{*}+D_{21} D_{21}^{*}\right)^{-1}\left(D_{21} \Gamma_{1}^{*}+C_{2} Q \Phi^{*}\right)+\tau_{\perp} \tilde{Q}_{\perp}^{*}, \\
& P=\Phi^{*} P \Phi+C_{1}^{*} C_{1} \\
& -\left(C_{1}^{*} D_{12}+\Phi^{*} P \Gamma_{2}\right)\left(\Gamma_{2}^{*} P \Gamma_{2}+D_{12}^{*} D_{12}\right)^{-1}\left(D_{12}^{*} C_{1}+\Gamma_{2}^{*} P \Phi\right)+\tau_{\perp}^{*} \bar{P}_{\perp}, \\
& \bar{Q}=\left[\Phi-\Gamma_{2}\left(\Gamma_{2}^{*} P \Gamma_{2}+D_{12}^{*} D_{12}\right)^{-1}\left(\Gamma_{2}^{*} P \Phi+D_{12}^{*} C_{1}\right)\right] \tau \bar{Q} \tau^{*} \text {. } \\
& \cdot\left[\Phi-\Gamma_{2}\left(\Gamma_{2}^{*} P \Gamma_{2}+D_{12}^{*} D_{12}\right)^{-1}\left(\Gamma_{2}^{*} P \Phi+D_{12}^{*} C_{1}\right)\right]^{*} \\
& +\left(\Phi Q C_{2}^{*}+\Gamma_{1} D_{21}^{*}\right)\left(C_{2} \dot{Q} C_{2}^{*}+D_{21} D_{21}^{*}\right)^{-1}\left(C_{2} Q \Phi^{*}+D_{21} \Gamma_{1}^{*}\right), \\
& \bar{P}=\left[\Phi-\left(\Phi Q C_{2}^{*}+\Gamma_{1} D_{21}^{*}\right)\left(C_{2} Q C_{2}^{*}+D_{21} D_{21}^{*}\right)^{-1} C_{2}\right]^{*} \tau^{*} \bar{P} \tau \text {. } \\
& \cdot\left[\Phi-\left(\Phi Q C_{2}^{*}+\Gamma_{1} D_{21}^{*}\right)\left(C_{2} Q C_{2}^{*}+D_{21} D_{21}^{*}\right)^{-1} C_{2}\right] \\
& +\left(\Phi^{*} P \Gamma_{2}+C_{1}^{*} D_{12}\right)\left(\Gamma_{2}^{*} P \Gamma_{2}+D_{12}^{*} D_{12}\right)^{-1}\left(\Gamma_{2}^{*} P \Phi+D_{12}^{*} C_{1}\right),
\end{aligned}
$$

Proof. For a proof of Theorem 2, the reader is referred to [4].

The solution to the digital LQG control of Pritchard-Salamon systems can be obtained by the following procedure.

\section{Algorithm}

Step 1. Construct the equivalent discrete-time LQG control problem for the lifted Pritchard-Salamon system as in Theorem 1.

Step 2. Check for nuclearity of $\Gamma_{1} \Gamma_{1}^{*}$ and $C_{1}^{*} C_{1}$.

Step 3. Calculate the controllability and observability gramians of the closed-loop system and check for the invertibility of $D_{21} D_{21}^{*}+C_{2} Q C_{2}^{*}$ and $D_{12}^{*} D_{12}+\Gamma_{2}^{*} P \Gamma_{2}$.

Step 4. If the checks for nuclearity and invertibility in Steps 2 and 3 are positive, then construct the $n$-dimensional compensator as provided by Theorem 2 ; otherwise stop. 


\section{Conclusions}

In this paper, we have set out a Hyland-Bernstein approach to the fixed finite-order digital control of Pritchard-Salamon infinite-dimensional systems with unbounded input and output operators. The solutions implies the solvability of a discrete-time Hyland-Bernstein system of equations (two modified Riccati equations coupled with two modified Lyapunov equations via an oblique projection operator). The natural continuation of this work is the task of implementation.

\section{REFERENCES}

1. Balakrishnan, A. V. 1981. Applied functional analysis (2nd edn, vol. 3). Springer.

2. Bamieh, B. A., \& Pearson JR., J. B. 1993. A general framework for linear periodic systems with applications to $\mathcal{H}^{\infty}$ sampled-data control. IEEE Trans AC 37, 418-35.

3. BARB, F. D., DE KonING, W. L., \& Weiss, M. 1993. The digital linear quadratic control problem for Pritchard-Salamon systems. Proceedings of the 2nd European Control Conference ECC'93. Groningen, The Netherlands, 1993. Pp. 1371-76.

4. Barb F. D., \& DE KonING, W. L. 1993. Necessary conditions for fixed, finite-order $\ell^{2}-$ optimal control of discrete-time evolution systems. Proceedings of the 32nd IEEE Conference on Decision and Control, San Antonio, 1993.

5. Bernstein, D. S., Davis, L. D., \& Hyland, D. C. 1986. The optimal projection equations for reduced-order discrete-time modeling, estimation and control. J. Guidance 9, 288-93.

6. Bernstein, D. S., \& Hyland, D. C. 1986. The optimal projection equations for finitedimensional fixed-order dynamic compensation of infinite-dimensional systems. SIAM J. Control \& Optimiz. 24, 122-51.

7. BernSTEIN, D. S., \& HADDAD, W. M. 1987. Optimal projection equations for discrete-time fixed-order dynamic compensation of linear systems with multiplicative white noise. Int.J. Control 46, 65-73.

8. Bontsema, J., \& Curtain, R. 1988. Perturbation properties of a class of infinite-dimensional systems with unbounded control and observation. IMA J. Math. Control \& Info. $5,333-52$.

9. Curtain, R., Logemann, H., Townley, S., \& Zwart, H. 1992. Well-posedness, stabilizability and admissibility for Pritchard-Salamon systems. Report No. 260-1 of the Institute of Dynamical Systems, University of Bremen.

10. Pritchard, A. J., \& Salamon, D. 1985. The linear quadratic control problem for retarded systems with delays in control and observation. IMA J. Math. Control \& Info 2, 335-62.

11. Pritchard, A. J., \& Salamon, D. 1987. The linear quadratic optimal control problem for infinite-dimensional systems with unbounded input and output operators. SIAM J. Control \& Optimiz. 25, 121-44.

12. VAN KeULEN, B. 1993. $\mathcal{H}^{\infty}$-control for infinite-dimensional systems: a state space approach. Ph.D. Thesis, University of Groningen.

13. Үамамото, Y. 1990. New approach to sampled-data control systems. A function space method. Proceedings of the 29th Conference on Decision and Control, Honolulu, Hawai, 1990. Pp. 1882-7. 
